\title{
History of Diabetic Eye Related Diseases
}

\author{
Christy E. Benson ${ }^{1}$, Swathi Somisetty', Hannah M. I. Martin ${ }^{3}$ \\ ${ }^{1}$ Broadlawns Medical Center, Des Moines, USA \\ ${ }^{2}$ Department of Ophthalmology, Stein Eye Institute, University of California Los Angeles, Los Angeles, USA \\ ${ }^{3}$ Department of Medicine, University of Missouri, Kansas City, USA \\ Email: cbenson@broadlawns.org
}

How to cite this paper: Benson, C.E., Somisetty, S. and Martin, H.M.I. (2021) History of Diabetic Eye Related Diseases. Journal of Diabetes Mellitus, 11, 354-358. https://doi.org/10.4236/jdm.2021.115028

Received: September 30, 2021

Accepted: November 15, 2021

Published: November 18, 2021

Copyright $\odot 2021$ by author(s) and Scientific Research Publishing Inc. This work is licensed under the Creative Commons Attribution International License (CC BY 4.0).

http://creativecommons.org/licenses/by/4.0/

\section{(c) (i) Open Access}

\begin{abstract}
Diabetes mellitus is the number one cause of blindness among adults aged 20 64 in the United States. The Center for Disease Control estimates that 9.4\% of the US population meets diagnostic criteria for diabetes. Among patients with the disease, over one-fourth will have findings of diabetic retinopathy, ranging from mild non-proliferative diabetic retinopathy (rare dot-blot hemorrhages) to the most severe form, proliferative diabetic retinopathy (PDR). Despite diabetes being recognized as a unique disease entity as early as the 2nd century $\mathrm{AD}$, no physician was capable of identifying the connection between this mysterious endocrine disorder and its ocular manifestations until the 19th century, mainly due to limitations in the ocular examination. As imaging modalities have developed over the decades of research, so have our various treatments; which now include a wide range of options such as anti-VEGF injections, laser treatment, and microsurgeries. Here we review the evolution of imaging modalities, pathophysiology of proliferative vs. non-proliferative diabetic retinopathy complications, and commonly used treatment options.
\end{abstract}

\section{Keywords}

Diabetes Mellitus, Ocular Manifestations, Diabetic Retinopathy

\section{Introduction}

Proliferative diabetic retinopathy (PDR) is a microvascular disease mediated by Vascular Endothelial Growth Factor (VEGF), which is produced in response to retinal ischemia. The VEGF family consists of signaling protein isoforms that are required for normal vascular development. Human studies have shown that eyes with PDR have an abnormally high level of VEGF in the vitreous as compared to unaffected eyes. When VEGF is produced in excess, abnormal angiogenesis is triggered and creates neovascular membranes that are prone to leak. This lea- 
kage is caused by disruption of endothelial tight-junctions and the development of endothelial cell fenestration. This abnormal vascular permeability is what ultimately leads to retinal exudates and diabetic macular edema. In PDR, this neovascularization can also extend into the anterior segment and cause other ocular manifestations of diabetes such as neovascularization of the iris (NVI) and neovascularization of the angle (NVA), which ultimately leads to neovascular glaucoma (NVG). Long-standing hyperglycemia can also cause other ocular complications such as cataracts, ophthalmoplegia, and non-arteritic ischemic optic neuropathy (NAION) [1].

\section{Discussion}

In 1851, the invention of the direct ophthalmoscope opened the door for in vivo fundoscopy. As a result of this invention, ocular manifestations of systemic diseases could be identified and classified for the first time. Five years after the invention of the direct ophthalmoscope, Austrian physician Eduard Jäger described the increased incidence of retinal exudates in diabetic eyes. In his paper entitled "Beiträge zum Pathologie des Auges", he included twenty fundus drawings, and made the first claims that there was a direct correlation. In dispute with Jäger's observation, German physician Albrecht von Graefe argued that Jäger could not prove causality between diabetes and retinal vascular complications such as retinal exudates. Unfortunately, the belief that diabetes caused vascular changes in the retina remained controversial for the next two decades due to a lack of histopathologic proof; however, by 1872, British ophthalmologist Edward Nettleship confirmed Jäger's theory in his landmark paper entitled, "on oedema or cystic disease of the retina". This paper provided the first histopathological proof of cystoid macular changes in patients with diabetes. This was followed by multiple other ophthalmologists, such as Wilhelm Manz, who wrote about his observations of ocular manifestations of diabetes such as tractional retinal detachments and vitreous hemorrhages in his paper entitled "retinitis proliferans". Despite Jäger's and Nettleship's publications, the debate persisted into the early 20th century as to whether the macular findings were directly related to diabetes, or whether they were due to other vasculopathic risk factors that are commonly found in diabetics, such as atherosclerosis. In 1944, ophthalmologists Ballantyne and Lowenstein first used the term "diabetic retinopathy", and Scottish physician Arthur James Ballantyne also provided further evidence that diabetic retinopathy was a unique vasculopathy [1] [2].

In 1961, fluorescein angiography was introduced as an innovative modality to view vascular changes in the retina. This test, which is still commonly utilized, involves injecting a dye intravenously, followed by taking a rapid series of pictures to document the retinal blood flow and specific patterns of leakage. This advancement eventually led to the development of a formal classification system for diabetic retinopathy at the Airlie House meeting of 1968. By 1970, LASER photocoagulation had become widely used in the management of diabetic reti- 
nopathy, despite a lack of supporting evidence for its use. In 1971, the National Eye Institute (NEI) funded the Diabetic Retinopathy Study (DRS). The DRS, which was completed in 1975, was a landmark study that examined the effects of pan-retinal photocoagulation (PRP) through both xenon LASER and argon LASER in eyes with a minimum of severe non-proliferative diabetic retinopathy in both eyes. The goal of this study was to determine whether PRP was superior to observation alone. The study showed that LASER therapy was indeed beneficial to patients with PDR, and also showed that argon LASERs created fewer adverse effects than xenon LASERs while being equally effective. Furthermore, it was made clear in the study that half of eyes with PDR and high-risk characteristics will eventually progress to severe vision loss (visual acuity $<5 / 200$ ) without LASER treatment. High risk characterizations were identified as: 1) presence of vitreous or preretinal hemorrhage, 2) presence of active neovascularization, 3) location of the neovascularization on or within one disc diameter of the optic disc, 4) neovascularization of the disc greater than $1 / 3$ of the disc area or neovascularization elsewhere greater than $1 / 2$ of the disc area. Eyes with high risk characteristics were found to reap the greatest benefit from PRP. No clear benefit was found in eyes with severe NPDR or PDR without high risk characteristics. Despite new therapies in the present day, PRP remains the mainstay of treatment for PDR fifty years after its efficacy was first published by the National Eye Institute [3] [4].

By 1970, Robert Machemer introduced the first closed system surgical access to the posterior segment of the eye. This enabled surgical removal of opacities in the vitreous, including vitreous hemorrhages, using microsurgical instrumentation through the pars plana. Three years later, Conor O'Malley and Ralph Heintz would develop the modern-day three-port vitrectomy system with dedicated ports for removal of the vitreous using a cutter, infusion cannula to maintain intraocular pressure, and illumination of the posterior segment with a light. The instruments used for vitrectomies were initially 20 -gauge in size, but further innovation led to the development of 27 -gauge instrumentation $(0.40 \mathrm{~mm}$ incision size) by 2010, further improving the safety profile of this commonly performed surgical procedure known as the Pars Plana Vitrectomy (PPV) [5].

By 1985, the Early Treatment Diabetic Retinopathy Study (ETDRS) would publish the data from its five year trial and would further standardize management strategies for diabetic retinopathy. Its goal was to evaluate the effectiveness of both argon LASER photocoagulation and aspirin therapy in delaying or preventing progression of the disease. Aspirin was found to have zero effect on the progression of retinopathy, but did reduce cardiovascular risk and did not increase the risk for vitreous hemorrhage. The study standardized the definition of "clinically significant macular edema (CSME)", and demonstrated a clear benefit of focal macular LASER [5] [6].

Modern day treatment of diabetic macular edema relies more heavily on the results of macular optical coherence tomography (OCT) than it does fluorescein 
angiography. The first in vivo OCT images of the retina were published in 1993, and gained widespread clinical use by the late 1990's. Optical coherence tomography is a light-based technique that provides tissue imagery at high resolutions and can yield great accuracy in the diagnosis of diabetic macular edema. It is a non-invasive imaging modality that is routinely performed to monitor DME progression at the molecular level and can help determine whether or not to initiate treatment for diabetic macular edema [4] [7].

The identification of VEGF as a major cause of macular edema and neovascularization led to the current use of intravitreal anti-vascular endothelial growth factor (VEGF) intravitreal injections to treat macular edema. Several studies have demonstrated that anti-VEGF therapy is more effective than focal LASER treatment for macular edema. Ranibizumab gained FDA approval in 2012 for the treatment of diabetic macular edema, followed by Aflibercept receiving FDA approval in 2014 [8].

Ophthalmoplegia is an entity which is less common than diabetic retinopathy but, as a result of the complications from both a diagnostic and treatment viewpoint, is often a source of anxiety for affected patients. In a study conducted over a ten-year period by "S. Biago" Hospital, Marsala, statistics were collected on diabetic patients who exhibited ophthalmoplegia. This study showed that while it is a serious condition, ophthalmoplegia is very uncommon in diabetic patients; it was identified in only $0.40 \%$ of patients. Furthermore, the study showed that out of all patients who exhibited ophthalmoplegia, 59.3\% was accounted for by isolated Cranial Nerve III palsies, followed by Cranial Nerve VI palsies (29.6\% of patients), and $11.1 \%$ of patients showed multiple palsies. Patients who exhibited symptoms of ophthalmoplegia in the study were largely found to have poorly controlled diabetes [9].

While diabetes has many ocular complications, recent years have brought many advancements in the areas of treatments and imaging modalities. These include antiVEGF injections, LASERs, and microsurgeries such as pars plana vitrectomy and cataract surgery. As a result of these modern treatments, many diabetic patients maintain usable vision for the duration of their lives [6].

\section{Conflicts of Interest}

The authors declare no conflicts of interest regarding the publication of this paper.

\section{References}

[1] Kalantzis, G., Angelou, M. and Poulakou-Rebelakou, E. (2006) Diabetic Retinopathy: A Historical Assessment. Hormones, 5, 72-75. https://doi.org/10.14310/horm.2002.11172

[2] Wolfensberger, T.J. and Hamilton, P. (2001) Diabetic Retinopathy-An Historical Review. Seminars in Ophthalmology, 16, 2-7. https://doi.org/10.1076/soph.16.1.2.4220

[3] Matuszewski, W., Bandurska-Stankiewicz, E., Modzelewski, R., Kamińska, U. and 
Stefanowicz-Rutkowska, M. (2017) Diagnosis and Treatment of Diabetic Retinopathy-Historical Overview. Clinical Diabetology, 6, 182-188.

https://doi.org/10.5603/DK.2017.0030

[4] Fine, S.L., Goldberg, M.F. and Tasman, W. (2016) Historical Perspectives on the Management of Macular Degeneration, Diabetic Retinopathy, and Retinal Detachment. American Academy of Ophthalmology, 123, S64-S77. https://doi.org/10.1016/j.ophtha.2016.04.058

[5] Mansour, S.E., Browning, D.J., Wong, K., Flynn Jr, H.W. and Bhavsar, A.R. (2020) The Evolving Treatment of Diabetic Retinopathy. Clinical Ophthalmology, 14, 653-678. https://doi.org/10.2147/OPTH.S236637

[6] Relhan, N. and Flynn Jr, H.W. (2017) The Early Treatment Diabetic Retinopathy Study Historical Review and Relevance to Today's Management of Macular Edema. Current Opinion in Ophthalmology, 28, 205-212.

https://doi.org/10.1097/ICU.0000000000000362

[7] Nguyen, Q.D., Brown, D.M., Marcus, D.M., Boyer, D.S., Patel, S., Feiner, L., Gibson, A., Sy, J., Rundle, A.C., Hopkins, J.J., Rubio, R.G. and Ehrlich, J.S. (2012) Ranibizumab for Diabetic Macular Edema. American Academy of Ophthalmology, 119, 789-801. https://doi.org/10.1016/j.ophtha.2011.12.039

[8] Brown, D.M., Nguyen, Q.D., Marcus, D.M., Boyer, D.S., Patel, S., Feiner, L., Schlottmann, P.G., Rundle, A.C., Zhang, J., Rubio, R.G., Adamis, A.P., Ehrlich, J.S. and Hopkins, J.J. (2013) Long-Term Outcomes of Ranibizumab Therapy for Diabetic Macular Edema: The 36-Month Results from Two Phase III Trials. American Academy of Ophthalmology, 120, 2013-2022. https://doi.org/10.1016/j.ophtha.2013.02.034

[9] Greco, D., Gambina, F. and Maggio, F. (2009) Ophthalmoplegia in Diabetes Mellitus: A Retrospective Study. Acta Diabetologica, 46, Article No. 23.

https://doi.org/10.1007/s00592-008-0053-8 